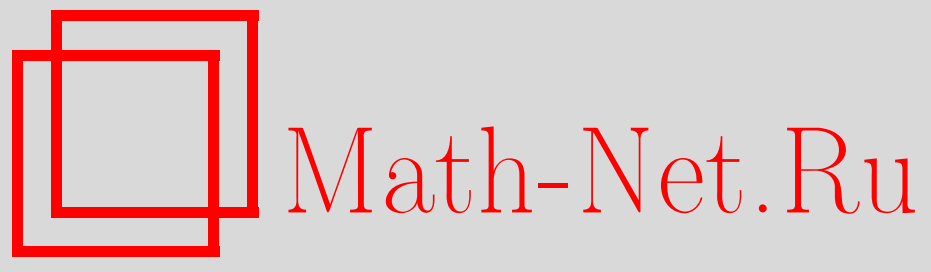

Ю. М. Воробьев, Линеаризуемость пуассоновых структур на сингулярных симплектических листах, Матем. заметки, 2006, том 80, выпуск 6, 825-837

DOI: https://doi.org/10.4213/mzm3360

Использование Общероссийского математического портала Math-Net.Ru подразумевает, что вы прочитали и согласны с пользовательским соглашением http://www . mathnet.ru/rus/agreement

Параметры загрузки:

IP: 3.91 .87 .62

26 апреля 2023 г., 17:43:17

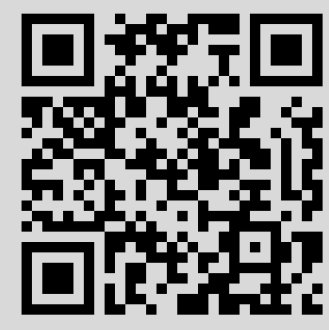




\section{ЛИНЕАРИЗУЕМОСТЬ ПУАССОНОВЫХ СТРУКТУР \\ НА СИНГУЛЯРНЫХ СИМПЛЕКТИЧЕСКИХ ЛИСТАХ}

\section{Ю. М. Воробьев}

Изучается задача о линеаризации пуассоновой структуры вблизи сингулярного симплектического листа ненулевой размерности. Получено следующее обобщение теоремы линеаризации Конна: если трансверсальная алгебра Ли листа полупроста и компактна, то пуассонова структура становится линеаризуемой при обращении в нуль некоторых когомологических препятствий.

Библиография: 15 названий.

\section{1. Введение}

Задача о линеаризации [1], [2] пуассоновых структур вблизи сингулярных точек (симплектических листов нулевой размерности) изучалась в многочисленных работах; см., например, [3]-[6]. Известная теорема линеаризации, полученная в [3], гласит, что если трансверсальная алгебра Ли g некоторой сингулярной точки пуассонова многообразия проста и компактна, то пуассонова структура локально изоморфна структуре Ли-Пуассона на $\mathfrak{g}^{*}$.

Целью настоящей работы является изучение задачи о линеаризации данной пуассоновой структуры $\Psi$ вблизи (сингулярного) симплектического листа $B$ ненулевой размерности в рамках теории пуассоновых структур спаривания [7]-[10]. В работе [7] было введено понятие линеаризированной пуассоновой структуры для $\Psi$ над симплектическим листом $B$. Здесь мы получаем следующее обобщение теоремы Конна: если трансверсальная алгебра Ли листа $B$ полупроста и компактна, то ростки структуры $\Psi$ на листе $B$ и линеаризированная пуассонова структура являются изоморфными при обращении в нуль некоторых когомологических препятствий. Эти препятствия связаны с понятием относительного коцикла Казимира, соответствующего паре пуассоновых структур спаривания [9]. Примеры нелинеаризуемых пуассоновых структур можно найти в [9], [11]. Различные аспекты задачи линеаризации в случае ненулевой размерности также обсуждаются в [12], [13].

\section{2. Основные результаты}

Чтобы сформулировать основные результаты, мы сначала напомним некоторые факты относительно пуассоновых тензоров спаривания (более подробно см. [7]-[10]).

Работа выполнена при частичной поддержке фонда CONACYT, грант № 43208.

(C) Ю. М. Воровьев, 2006 
На протяжении всей статьи по повторяющимся индексам будет подразумеваться суммирование.

2.1. Пуассоновы тензоры спаривания. Пусть $(M, \Psi)$ - пуассоново многообразие со скобкой Пуассона $\{F, G\}_{\Psi}=\left\langle d G, \Psi^{\natural} d F\right\rangle$. Здесь $\Psi^{\natural}: T^{*} M \rightarrow T M-$ морфизм векторных расслоений, порожденный тензором Пуассона $\Psi \in \chi^{2}(M)$. Предположим, что задан вложенный симплектический лист $(B, \omega)$ пуассоновой структуры $\Psi$.

Рассмотрим нормальное расслоение $\pi: E \rightarrow B$ листа $B, E=T_{B} E / T B$. Конормальное расслоение $E^{*}$ несет естественную структуру локально тривиального расслоения алгебр Ли над $B$, индуцированного исходной пуассоновой структурой. Типичный слой $\mathfrak{g}$ этого расслоения называется трансверсальной алгеброй Ли симплектического листа $B$ в $(M, \Psi)$. Тогда нормальное расслоение $E$ становится локально тривиальным расслоением многообразий Ли-Пуассона с типичным слоем $\mathfrak{g}^{*}$.

Рассмотрим естественное разложение $T_{B} E=T B \oplus E$. Под экспоненциалъным отображением будем понимать диффеоморфизм $f: E \rightarrow M$ из тотального пространства на окрестность листа $B$ в $M$, удовлетворяющий следующим условиям:

$$
\left.\boldsymbol{f}\right|_{B}=\operatorname{id}_{B} \quad \text { и } \quad T_{B} M=T B \oplus d_{B} \boldsymbol{f}(E) .
$$

Из теоремы о трубчатой окрестности следует, что для любого подрасслоения $\mathscr{L}$, являющегося дополнением к $T B$ в $T_{B} M$, существует экспоненциальное отображение такое, что $\mathscr{L}=d_{B} \boldsymbol{f}(E)$.

Пусть $\boldsymbol{f}: E \rightarrow M$ и $\widetilde{\boldsymbol{f}}: E \rightarrow M$ - два экспоненциальных отображения. Тогда диффеоморфизм $\varphi=\widetilde{\boldsymbol{f}}^{-1} \circ \boldsymbol{f}$ между двумя окрестностями $B$ в $E$ имеет следующие свойства:

$$
\begin{gathered}
\left.\varphi\right|_{B}=\operatorname{id}_{B}, \\
\left.d_{b} \varphi\right|_{E_{b}}=\operatorname{id}_{E_{b}} \quad \forall b \in B .
\end{gathered}
$$

Группу ростков на $B$ всех диффеоморфизмов $\varphi$, удовлетворяющих условиям (2.1), $(2.2)$, будем обозначать через $\mathfrak{G}_{B}(E)$. Через $\mathfrak{G}_{B}^{\mathrm{fib}}(E)$ обозначим подгруппу в $\mathfrak{G}_{B}(E)$, состоящую из ростков на $B$ диффеоморфизмов, сохраняющих слои.

Зафиксируем экспоненциальное отображение $\boldsymbol{f}_{0}$. Ясно, что росток на $B$ любого экспоненциального отображения $\boldsymbol{f}$ отличается от ростка заданного экспоненциального отображения $\boldsymbol{f}_{0}$ на некоторый элемент из $\mathfrak{G}_{B}(E)$.

Пусть $\Pi=\boldsymbol{f}_{0}^{*} \Psi$ - поднятие исходного тензора Пуассона $\Psi$ при отображении $\boldsymbol{f}_{0}$ на тотальное пространство $E$. Заметим, что $B$ (как нулевое сечение расслоения $\pi$ ) является симплектическим листом пуассоновой структуры П. Пусть $E_{U} \approx U \times \mathbb{R}^{r}-$ локальная тривиализация нормального расслоения $\pi$, где $U$ является открытой картой листа $B$ с локальными координатами $\xi=\left(\xi^{i}\right)$. Пусть $x=\left(x^{\sigma}\right)-$ координаты вдоль слоев $\pi$, соответствующие некоторому базису сечений расслоения $E$. Тогда $B$ локально определяется уравнениями $x^{1}=\cdots=x^{r}=0$. В терминах локальной системы координат $(\xi, x)=\left(\xi^{i}, x^{\sigma}\right)$, скобка Пуассона, соответствующая бивекторному полю П, задается соотношениями

$$
\begin{gathered}
\left\{\xi^{i}, \xi^{j}\right\}_{\Pi}=-F^{i j}(\xi, x), \\
\left\{\xi^{i}, x^{\sigma}\right\}_{\Pi}=F^{i s}(\xi, x) \Gamma_{s}^{\sigma}(\xi, x), \\
\left\{x^{\alpha}, x^{\beta}\right\}_{\Pi}=\mathscr{V}^{\alpha \beta}(\xi, x)-\Gamma_{i}^{\alpha}((\xi, x)) F^{i j}(\xi, x) \Gamma_{j}^{\beta}(\xi, x) .
\end{gathered}
$$


Здесь $F^{i j}, \Gamma_{i}^{\alpha}$ и $\mathscr{V}^{\alpha \beta}$ - гладкие (локальные) функции на $E$. Так как $B$ является симплектическим листом структуры П, то

$$
\Gamma_{s}^{\sigma}(\xi, 0)=0, \quad \mathscr{V}^{\alpha \beta}(\xi, 0)=0 .
$$

Пусть

$$
\omega=\frac{1}{2} \omega_{i j}(\xi) d \xi^{i} \wedge d \xi^{j}
$$

- симплектическая форма на $B$. Ясно, что $F^{i j}(\xi, 0)=\omega^{i j}(\xi)$, где $\omega^{i s}(\xi) \omega_{s j}(\xi)=\delta_{j}^{i}$. Так как матрица $\left(\omega^{i j}(\xi)\right)$ является невырожденной, то для любой точки $b \in B$ можно зафиксировать открытую окрестность $N_{b}$ точки $b$ в $E$ так, чтобы $N_{b} \subset E_{U}$ и

$$
\left.\operatorname{det}\left(F^{i j}(\xi, x)\right)\right) \neq 0 \quad \forall(\xi, x) \in N_{b} .
$$

Тогда $N=\bigcup_{b \in B} N_{b}$ является открытой окрестностью $B$ в $E$.

Пусть $\mathbf{V}=\operatorname{ker} d \pi \subset T E$ - вертикальное подрасслоение, и пусть Ann $\mathbf{V}$ - аннулятор этого подрасслоения. Введем

$$
\mathbf{H} \stackrel{\text { def }}{=} \Pi^{\natural}(\text { Ann } \mathbf{V}) \subset T_{N} E .
$$

Из условия невырожденности (2.7) следует, что Н является дополнением к V,

$$
T_{N} E=\mathbf{H} \oplus \mathbf{V} .
$$

Таким образом, горизонтальное подрасслоение $\mathbf{H}$ определяет каноническую связность Эресмана, соответствующую пуассоновой структуре П. Пусть Г - соответствующая форма связности. В координатной записи имеем

$$
\Gamma=\left(d x^{\nu}+\Gamma_{i}^{\nu}(\xi, x) d \xi^{i}\right) \otimes \frac{\partial}{\partial x^{\nu}},
$$

где коэффициенты $\Gamma_{i}^{\nu}$ задаются соотношением (2.4). Горизонтальный лифт базисного векторного поля $\partial / \partial \xi^{i}$ имеет вид

$$
\operatorname{hor}_{i}^{\Gamma}=\frac{\partial}{\partial \xi^{i}}-\Gamma_{i}^{\nu}(\xi, x) \frac{\partial}{\partial x^{\nu}},
$$

и $\operatorname{hor}^{\Gamma}(u)=u^{i}(\xi) \operatorname{hor}_{i}$ для любого векторного поля $u=u^{i}(\xi)\left(\partial / \partial \xi^{i}\right)$ на $B$.

Тензорное поле на $N$ называется горизонтальным или вертикальным, если оно касательно соответственно к $\mathbf{H}$ или V. С помощью функций $\mathscr{V} \alpha \beta$, заданных в (2.5), вводится следующее вертикальное бивекторное поле $\mathscr{V}$ на $N$ :

$$
\mathscr{V}=\mathscr{V}^{\alpha \beta}(\xi, x) \frac{\partial}{\partial x^{\alpha}} \wedge \frac{\partial}{\partial x^{\beta}},
$$

которое обращается в нуль вдоль $B$,

$$
\operatorname{rank} \mathscr{V}=0 \text { на } B
$$

В силу (2.5) и тождества Якоби для переменных $\left(x^{\alpha}\right)$ легко увидеть, что $\mathscr{V}$ является тензором Пуассона. Для любой $\xi \in B$ сужение $\mathscr{V}$ на слой $N \cap E_{\xi}$ задает трансверсальную пуассонову структуру для П в точке $\xi[2]$. Мы также будем говорить, что $\mathscr{V}$ - это трансверсальная пуассонова структура листа $B$. 
В общем случае расщепление (2.9) индуцирует разложение любого бивекторного поля в сумму 2-тензорных полей типа $(2,0),(0,2)$ и $(1,1)$. Компоненты степени $(2,0)$ и $(0,2)$ соответствуют горизонтальному и вертикальному бивекторному полю соответственно.

ПРЕДЛОЖЕНИЕ 2.1. В окрестности $N$ листа В пуассонов тензор П допускает разложение

$$
\Pi=\Pi_{H}+\mathscr{V},
$$

где $\mathscr{V}$ - трансверсальный пуассонов тензор в (2.12) и $\Pi_{H}$ - горизонтальное бивекторное поле, задаваемое соотношением

$$
\Pi_{H}=-\frac{1}{2} F^{i j}(\xi, x) \operatorname{hor}_{i}^{\Gamma} \wedge \operatorname{hor}_{j}^{\Gamma} .
$$

Кроме того, $\Pi_{H}$ является пуассоновым тензором тогда и только тогда, когда кривизна связности Г равна нулю.

Будем отождествлять пространство горизонтальных $k$-форм на $E$ с пространством $\Omega^{k}(B) \otimes C^{\infty}(E)$ векторнозначных $k$-форм на $B$. Напомним, что $\Gamma$-ковариантная внешняя производная

$$
\partial^{\Gamma}: \Omega^{k}(B) \otimes C^{\infty}(E) \rightarrow \Omega^{k+1}(B) \otimes C^{\infty}(E)
$$

определяется как

$$
\begin{aligned}
\left(\partial^{\Gamma} \Theta\right)\left(u_{0}, u_{1}, \ldots, u_{k}\right) \stackrel{\text { def }}{=} & \sum_{i=0}^{k}(-1)^{i} L_{\mathrm{hor}^{\Gamma}\left(u_{i}\right)} \Theta\left(u_{0}, u_{1}, \ldots, \widehat{u}_{i}, \ldots, u_{k}\right) \\
& +\sum_{0 \leqslant i<j \leqslant k}(-1)^{i+j} \Theta\left(\left[u_{i}, u_{j}\right], u_{0}, u_{1}, \ldots, \widehat{u}_{i}, \ldots, \widehat{u}_{j}, \ldots, u_{k}\right) .
\end{aligned}
$$

Пусть $\operatorname{Curv}^{\Gamma}$ - форма кривизны связности Г. Введем горизонтальную 2-форму

$$
F=\frac{1}{2} F_{i j}(\xi, x) d \xi^{i} \wedge d \xi^{j}
$$

на $N$, где $F_{i s}(\xi, x) F^{s j}(\xi, x)=\delta_{i}^{j}$ и $F^{s j}(\xi, x)$ заданы в (2.3). Тогда тождество Якоби для бивекторного поля (2.14) влечет следующие уравнения для данных $(\mathscr{V}, \Gamma, F)$ (см. [7]):

$$
\begin{gathered}
{[\mathscr{V}, \mathscr{V}]=0,} \\
L_{\operatorname{hor}^{\Gamma}(v)^{\mathscr{V}}=0,} \\
\partial^{\Gamma} F=0, \\
\operatorname{Curv}^{\Gamma}(v, u)=(\mathscr{V})^{\sharp} d(F(v, u))
\end{gathered}
$$

для любых $v, u \in \mathscr{X}(B)$. Здесь $[\cdot, \cdot]$ - скобка Схоутена на $E$ [14], [15]. В частности, (2.17) означает, что горизонтальный лифт $\operatorname{hor}^{\Gamma}(v)$ является пуассоновым векторным полем вертикального пуассонова тензора $\mathscr{V}$.

Обратно, пусть задан триплет $(\mathscr{V}, \Gamma, F)$ удовлетворяющий уравнениям (2.16)(2.19) и такой, что 2-форма $F$ является невырожденной в смысле $(2.7)$. Тогда 
формула (2.14) определяет бивекторное поле П, которое называется пуассоновым тензором спаривания, соответствующим данным $(\mathscr{V}, \Gamma, F)$. Форма $F$ также называется формой спаривания. Таким образом, формула (2.14) является результатом спаривания $F$ и $\mathscr{V}$ с помощью связности $Г$.

Важным следствием предложения 2.1 является то, что каждая пуассонова структура на $E$ в окрестности симплектического листа $(B, \omega)$ реализуется как пуассонов тензор спаривания.

2.2. Линеаризированные пуассоновы структуры. Чтобы определить линеаризированную пуассонову структуру пуассонова тензора П на $B$, мы сначала применим процедуру линеаризации к данным $(\mathscr{V}, \Gamma, F)$. В результате, получим триплет $\left(\Lambda, \Gamma^{(1)}, F^{(1)}\right)$, который снова удовлетворяет соотношениям $(2.16)-(2.19)$ и, следовательно, определяет пуассонов тензор спаривания $\Pi^{(1)}$.

Принимая во внимание (2.6), мы получим следующие разложения Тейлора в точке $x=0$ :

$$
\begin{gathered}
F^{i j}(\xi, x)=-\omega^{i j}(\xi)-\omega^{i s} \mathscr{R}_{s m \nu} \omega^{m j} x^{\nu}+O\left(x^{2}\right), \\
\Gamma_{i}^{\sigma}(\xi, x)=\theta_{i \nu}^{\sigma}(\xi) x^{\nu}+O\left(x^{2}\right), \\
\mathscr{V}^{\alpha \beta}(\xi, x)=\lambda_{\gamma}^{\alpha \beta}(\xi) x^{\gamma}+O\left(x^{2}\right) .
\end{gathered}
$$

Используя "инфинитезимальные" данные $\theta_{i \nu}^{\sigma}(\xi), \lambda_{\gamma}^{\alpha \beta}(\xi)$, можно ввести

- связность Эресмана на $E$ :

$$
\Gamma^{(1)}=\theta_{i \alpha}^{\nu}(\xi) x^{\alpha} d \xi^{i} \otimes \frac{\partial}{\partial x^{\nu}},
$$

- вертикальное бивекторное поле $\Lambda$ на $E$ :

$$
\Lambda=\frac{1}{2} \lambda_{\gamma}^{\alpha \beta}(\xi) x^{\gamma} \frac{\partial}{\partial x^{\alpha}} \wedge \frac{\partial}{\partial x^{\beta}} .
$$

Заметим, что связность $\Gamma^{(1)}$ является однородной в том смысле, что производная Ли вдоль $\Gamma^{(1)}$-горизонтального лифта hor ${ }^{\Gamma^{(1)}}(u)$ сохраняет пространство послойно линейных функций на $E$. Это означает, что $\Gamma^{(1)}$ однозначно определяется линейной связностью $\nabla$ на $E$, которая задается соотношением $\nabla_{\partial / \partial \xi^{i}} X_{\sigma}=\theta_{i \sigma}^{\nu} X_{\nu}$. Здесь $\left\{X_{\sigma}\right\}$ - базис локальных сечений $E$, соответствующий координатам $\left(x^{\sigma}\right)$. Из тождества Якоби и разложения (2.22) следует, что $\Lambda$ - это тензор Пуассона на $E$, называемый линеаризированной трансверсальной пуассоновой структурой листа $B$. Эта структура не зависит от выбора экспоненциального отображения. Сужение $\Lambda$ на каждый слой $E_{\xi}$ изоморфно структуре Ли-Пуассона типичного слоя $\mathfrak{g}^{*}$, a $\lambda_{\gamma}^{\alpha \beta}(\xi)$ являются структурными константами для $E_{\xi}^{*}$, соответствующими дуальному базису $\left\{\eta^{\nu}\right\},\left\langle\eta^{\nu}, X_{\sigma}\right\rangle=\delta_{\sigma}^{\nu}$.

Теперь с помощью функций $\mathscr{R}_{s m \nu}$ в разложении (2.20) мы определим следующую векторнозначную 2-форму на $B$ :

$$
\mathscr{R}=\mathscr{R}_{\nu} \otimes \eta^{\nu}, \quad \mathscr{R}_{\nu}=\frac{1}{2} \mathscr{R}_{i j \nu}(\xi) d \xi^{i} \wedge d \xi^{j} .
$$

Линеаризированная 2-форма спаривания

$$
F^{(1)}=\frac{1}{2} F_{i j}^{(1)}(\xi, x) d \xi^{i} \wedge d \xi^{j}
$$


на $E$ задается соотношением

$$
F_{i j}^{(1)}(\xi, x)=\omega_{i j}(\xi)-\mathscr{R}_{i j \sigma}(\xi) x^{\sigma}
$$

Ясно, что можно выбрать окрестность $N$ так, чтобы матрица $\left(F_{i j}^{(1)}(\xi, x)\right)$ была невырожденной для всех $(\xi, x) \in N$. Напомним, что через $\left(F^{(1)}\right)^{i j}$ мы обозначаем элементы матрицы, обратной к $\left(F_{i j}^{(1)}(\xi, x)\right)$, и

$$
\operatorname{hor}_{i}^{\Gamma^{(1)}}=\frac{\partial}{\partial \xi^{i}}-\theta_{i \alpha}^{\nu}(\xi) x^{\alpha} \frac{\partial}{\partial x^{\nu}}
$$

- это $\Gamma^{(1)}$-горизонтальный лифт векторного поля $\partial / \partial \xi^{i}$.

ПреДЛОЖЕНИЕ 2.2. (а) Инфинитезимальные даннъе $\left(\Lambda, \Gamma^{(1)}, F^{(1)}\right)$ порождают следующий пуассонов тензор спаривания на $N$ :

$$
\Pi^{(1)}=\Pi_{H}^{(1)}+\Lambda
$$

¿əe

$$
\Pi_{H}^{(1)}=-\frac{1}{2}\left(F^{(1)}\right)^{i j}(\xi, x) \operatorname{hor}_{i}^{\Gamma^{(1)}} \wedge \operatorname{hor}_{j}^{\Gamma^{(1)}} .
$$

(b) Росток тензора $\Pi^{(1)}$ на В не зависит от выбора экспоненциального отображения с точностью до некоторого дифбеоморфизма в $\mathfrak{G}_{B}(E)$.

Пункт (а) этого предложения 2.2 доказывается проверкой [7] того факта, что тройка $\left(\Lambda, \Gamma^{(1)}, F^{(1)}\right)$ удовлетворяет соотношениям (2.16)-(2.19). Заметим, что в терминах линейной связности $\nabla$ условия (2.17)-(2.19) можно переформулировать следующим образом: линейная связность $\nabla$ сохраняет послойную структуру ЛиПуассона нормального расслоения $\pi, \nabla^{*} \mathscr{R}=0$ и $\mathrm{Curv}^{\nabla}=-\mathrm{ad}^{*} \circ \mathscr{R}$. Здесь Curv ${ }^{\nabla}=$ $d \theta+\theta \wedge \theta-$ это форма кривизны связности $\nabla$, a $\mathrm{ad}^{*}$ обозначает косопряженный оператор на слоях расслоения $\pi$. Пуассонов тензор спаривания $\Pi^{(1)}$ в $(2.28)$, соответствующий данным $\left(\Lambda, \Gamma^{(1)}, F^{(1)}\right)$, называется линеаризированной пуассоновой структурой поля П на $B$.

На самом деле, можно показать, что $\Pi^{(1)}$ зависит только от дополнения $\mathscr{L}=$ $d_{B} \boldsymbol{f}_{0}(E)$ к $T B$. Из пункта (b) предложения 2.2 следует, что корректно определено понятие линеаризированной пуассоновой структуры исходного пуассонова тензора $\Psi$ на $B$ : ростки на $B$ линеаризированных пуассоновых структур, параметризованных трансверсальными подрасслоениями $\mathscr{L}$, являются изоморфными, но естественного представителя для этой линеаризированной структуры не существует. В разделе 3 мы даем набросок доказательства пункта (b); более подробно см. [7].

2.3. Задача о линеаризации. Теперь мы можем сформулировать задачу о пуассоновой линеаризации.

ОПРЕДЕЛЕНИЕ 2.3. Пуассонова структура $\Psi$ называется линеаризируемой на симплектическом листе $B$, если существует экспоненциальное отображение $\boldsymbol{f}$ такое, что

$$
f^{*} \Psi=\Pi^{(1)} .
$$


Итак, линеаризуемость пуассоновой структуры $\Psi$ на $B$ означает, что ростки структур $\Psi$ и $\Pi^{(1)}$ изоморфны при некотором экспоненциальном отображении. Условие эквивалентности (2.30) можно переформулировать следующим образом: для заданного экспоненциального отображения $\boldsymbol{f}_{0}$, прообраз $\Pi=\boldsymbol{f}_{0}^{*} \Psi$ изоморфен $\Pi^{(1)}$ при некотором диффеоморфизме в $\mathfrak{G}_{B}(E)$,

$$
\varphi^{*} \Pi=\Pi^{(1)}, \quad \varphi \in \mathfrak{G}_{B}(E) .
$$

В этом случае можно выбрать $\boldsymbol{f}$ в $(2.30)$ в виде $\boldsymbol{f}=\boldsymbol{f}_{0} \circ \varphi$.

Первый шаг решения задачи о линеаризации - это проверка эквивалентности трансверсальных пуассоновых структур $\mathscr{V}$ и $\Lambda$. Выберем точку $\xi^{0} \in B$ и рассмотрим слой $E_{\xi^{0}}$. Через $\operatorname{Diif}_{0}\left(E_{\xi^{0}}\right)$ обозначим группу ростков в точке 0 диффеоморфизмов на $E_{\xi^{0}}$, переводящих 0 в 0 . Рассмотрим сужения $\mathscr{V}_{\xi^{0}}=\left.\mathscr{V}\right|_{E_{\xi^{0}}}$ и $\Lambda_{\xi^{0}}=\left.\Lambda\right|_{E_{\xi^{0}}}$. Напомним, что $\mathscr{V}_{\xi^{0}}$ обращается в нуль в точке $0 \in E_{\xi^{0}}$. В соответствии со стандартным определением [2], [3] будем говорить, что пуассонова структура $\mathscr{V}_{\xi^{0}}$ линеаризуема в сингулярной точке, если ростки сужений $\mathscr{V}_{\xi^{0}}$ и $\Lambda_{\xi^{0}}$ в 0 изоморфны при некотором диффеоморфизме в $\operatorname{Diif}_{0}\left(E_{\xi^{0}}\right)$.

ПреДЛОЖЕНИЕ 2.4. Если $\mathscr{V}_{\xi^{0}}$ линеаризуемо в 0, то существует диффеоморфизм $g \in \mathfrak{G}_{B}^{\mathrm{fib}}(E)$ maкой, чmo

$$
g^{*} \mathscr{V}=\Lambda
$$

Другими словами, это утверждение гласит, что из линеаризуемости трансверсальной пуассоновой структуры в точке $\xi^{0}$ следует линеаризуемость вертикальной компоненты пуассонова тензора П на $B$. Доказательство предложения 2.4 основано на следующих двух фактах:

(i) трансверсальная структура $\mathscr{V}$ локально изоморфна прямому произведению нулевой пуассоновой структуры и $\mathscr{V}_{\xi^{0}}[2] ;$

(ii) каждый диффеоморфизм в $\operatorname{Diif}_{0}\left(E_{\xi^{0}}\right)$ может быть включен в поток некоторого векторного поля, зависящего от времени и обращающегося в нуль в 0 .

2.4. 2-коциклы Казимира. Здесь мы введем понятие 2-коцикла Казимира симплектического листа $B$, которое играет важную роль в наших рассмотрениях. Это понятие является корректным при некоторых дополнительных предположениях о трансверсальной алгебре Ли g листа $B$. Предположим, что

$$
\mathfrak{g} \text { полупроста и компактна. }
$$

ПРЕДЛОЖЕНИЕ 2.5. Если выполнено условие (2.33), то трансверсальная пуассонова структура $\mathscr{V}$ линеаризуема на $B$ в смысле (2.32).

Доказательство этого утверждения получается комбинированием предложения 2.4 и теоремы линеаризации Конна [3].

Теперь выберем диффеоморфизм $g \in \mathfrak{G}_{B}^{\mathrm{fib}}(E)$ в $(2.32)$ и рассмотрим тензор Пуассона $\widetilde{\Pi}=g^{*} \Pi$. Тогда имеем $\widetilde{\Pi}=\widetilde{\Pi}_{H}+\Lambda$, где $\widetilde{\Pi}_{H}=g^{*} \Pi_{H}$. Можно показать [7], [9], [10], что П Является пуассоновым тензором спаривания, соответствующим данным $(\Lambda, \widetilde{\Gamma}, \widetilde{F})$, где $\widetilde{\Gamma}=g^{*} \Gamma$ и $\widetilde{F}=g^{*} F-$ это поднятие связности $Г$ и формы спаривания $F$ с помощью диффеоморфизма $g$.

В соответствии с [3] при условии (2.33) каждое пуассоново векторное поле структуры Ли-Пуассона коалгебры $\mathfrak{g}^{*}$ на замкнутом шаре с центром в начале координат 
является гамильтоновым. Кроме того, существует оператор гомотопии комплекса Схоутена-Лихнеровича на $\mathfrak{g}^{*}$ для тензорных полей степеней 1 и 2 . Через $\mathfrak{F}_{B}$ обозначим пространство ростков на $B$ всех гладких функций, обращающихся в нуль на $B$. В силу вышеизложенного, принимая во внимание тот факт, что $\operatorname{hor}^{\widetilde{\Gamma}}(u)$ и $\operatorname{hor}^{\Gamma^{(1)}}(u)$ являются пуассоновыми векторными полями для $\Lambda$, мы получаем следующее утверждение.

ПрЕДЛОЖЕНИЕ 2.6. Существует 1-форма $Q \in \Omega^{1}(B) \otimes \mathfrak{F}_{B}$ такая, ито

$$
\operatorname{hor}^{\widetilde{\Gamma}}(u)=\operatorname{hor}^{\Gamma}(u)+\Lambda^{\natural} d Q(u)
$$

для любого $u \in \mathfrak{X}(B)$.

Через $\operatorname{Casim}_{B}(\Lambda)$ обозначим пространство всех функций Казимира для $\Lambda$, обращающихся в нуль на $B$. Соответствующее пространство ростков на $B$ элементов в $\operatorname{Casim}_{B}(\Lambda)$ будем обозначать через $\mathfrak{C a s i m}_{B}(\Lambda)$.

Рассмотрим ковариантную внешнюю производную $\partial^{\Gamma^{(1)}}$, соответствующую линеаризированной связности $\Gamma^{(1)}$. Так как $\operatorname{hor}^{\Gamma^{(1)}}(u)$ - это пуассоново векторное поле для $\Lambda$, сужение $\partial^{\Gamma^{(1)}}$ на $\Omega^{k}(B) \otimes \mathfrak{C a s i m}_{B}(\Lambda)$ определено корректно и порождает оператор

$$
\partial_{0}: \Omega^{k}(B) \otimes \mathfrak{C a s i m}_{B}(\Lambda) \rightarrow \Omega^{k+1}(B) \otimes \mathfrak{C a s i m}_{B}(\Lambda) .
$$

Из (2.34) и стандартного соотношения между $\left(\partial^{\Gamma^{(1)}}\right)^{2}$ и $\operatorname{Curv}^{\Gamma^{(1)}}$ [7], [9] следует, что $\partial_{0}$ является кограничным оператором, $\partial_{0}^{2}=0$. Можно показать, что при изменении экспоненциального отображения $\boldsymbol{f}_{0}$ связность $\Gamma^{(1)}$ будет меняться в соответствии с правилом перехода, аналогичным (2.34). Это означает, что $\partial_{0}$ не зависит от выбора $\boldsymbol{f}_{0}$. Фиксируя 1-форму $Q$ в $(2.34)$, введем следующую горизонтальную 2-форму на $B$ :

$$
C=C_{\widetilde{\Pi} \Pi^{(1)}} \stackrel{\text { def }}{=} \widetilde{F}-F^{(1)}+\left(\partial^{\Gamma^{(1)}} Q+\frac{1}{2}\{Q \wedge Q\}_{\Lambda}\right) .
$$

Здесь $\{\cdot, \cdot\}_{\Lambda}$ обозначает скобку Пуассона для $\Lambda$. Из тождества $(2.19)$ для $\widetilde{F}$ и $F^{(1)}$ следует, что $C \in \Omega^{2}(B) \otimes \mathfrak{C a s i m}_{B}(\Lambda)$.

2.5. Основные результаты. В предположении, что (2.33) выполняется, имеет место следующий важный результат.

Теорема 2.7. (а) Форма $C$ в (2.35) является 2-коциклом, $\partial_{0} C=0$.

(b) Класс $\partial_{0}$-когомологий $[C]$ не зависит от выбора диффеоморфизмов $\boldsymbol{f}_{0}, g u$ 1-формы $Q$ в (2.34).

Таким образом, класс когомологий $[C]$ является канонической характеристикой симплектического листа $B$ исходной пуассоновой структуры $\Psi$. Будем говорить, что $C$ - это 2-коиикл Казимира пары $\left(\widetilde{\Pi}, \Pi^{(1)}\right)$.

Теперь мы переходим к теореме о линеаризации.

Теорема 2.8. Если трансверсалъная алгебра Ли $\mathfrak{g}$ симплектического листа В полупроста и компактна, то пуассонова структура $\Psi$ линеаризуема на $B$ тогда $и$ только тогда, когда класс $\partial_{0}$-когомологий 2-коцикла Казимира $С$ нулевой,

$$
[C]=0 .
$$


Если условие (2.36) не выполняется, то будем говорить, что $\Psi$ нелинеаризуема на $B$.

ПримеР 2.9. Пусть $B$ - ориентированная поверхность в $\mathbb{R}^{3}$ с формой площади $\omega=d s \wedge d \tau$. Рассмотрим следующую скобку Пуассона на $E=B \times \mathbb{R}^{3}$ :

$$
\{s, \tau\}=\frac{1}{1+\|x\|^{2}}, \quad\left\{s, x_{\alpha}\right\}=\left\{\tau, x_{\alpha}\right\}=0, \quad\left\{x_{\alpha}, x_{\beta}\right\}=\varepsilon_{\alpha \beta \gamma} x_{\gamma} .
$$

Можно интерпретировать $E$ как тривиальное векторное расслоение над $B$. Тогда $(B, \omega)$ является симплектическим листом для (2.37) с трансверсальной алгеброй Ли $\mathfrak{g}=\mathrm{so}(3)$. Линеаризированная пуассонова структура совпадает с пуассоновой структурой прямого произведения на $B \times \mathrm{so}^{*}(3)$. Кограничный оператор $\partial_{0}$ порождается оператором $d_{B} \otimes$ id на $\Omega^{k}(B) \otimes C^{\infty}([0, \infty))$, где $d_{B}$ - внешняя производная на $B$. Коцикл Казимира задается как $C=\|\boldsymbol{x}\|^{2} \omega$. В случае, когда $B$ компактна, применяя теорему 2.8, мы заключаем, что пуассонова структура нелинеаризуема.

Другие примеры нелинеаризуемых пуассоновых структур можно найти в [9], [11]. Рассмотрим 2-коцикл Казимира (2.35)

$$
C=\frac{1}{2} C_{i j}(\xi, x) d \xi^{i} \wedge d \xi^{j}
$$

Тогда $C$ имеет нуль второго порядка на $B$, т.е. $C_{i j}=O\left(x^{2}\right)$. Введем деформированную форму спаривания $F_{C}^{(1)}=F^{(1)}+C$. Легко видеть, что данные $\left(\Lambda, \Gamma^{(1)}, F_{C}^{(1)}\right)$ удовлетворяют условиям (2.16)-(2.19) и, следовательно, порождают пуассонов тензор спаривания $\Pi_{C}$.

ТЕОРема 2.10. При условии (2.33) ростки на В пуассоновых структур $\Psi$ и $\Pi_{C}^{(1)}$ изоморбны.

\section{3. Метод гомотопии}

Здесь мы приведем доказательство основных результатов, сформулированных в разделе 2.

Пусть $\operatorname{Coup}(E, \Lambda)$ - множество всех пуассоновых тензоров спаривания П, определенных на некоторой окрестности $N$ листа $B$ в $E$ и удовлетворяющих условиям

$$
\begin{gathered}
(B, \omega)-\text { симплектический лист для } \Pi, \\
\Pi_{V}=\Lambda .
\end{gathered}
$$

Через $\mathfrak{C o u p}_{B}(E, \Lambda)$ обозначим множество ростков на $B$ бивекторных полей в $\operatorname{Coup}(E$, $\Lambda)$. Предположим, что задана гладкая кривая, состоящая из тензоров Пуассона

$$
[0,1] \ni t \mapsto \Pi_{t} \in \mathfrak{C o u p}_{B}(E, \Lambda)
$$

Для любого $t$ тензор $\Pi_{t}$ является пуассоновым тензором спаривания, соответствующим данным $\left(\Lambda, \Gamma_{t}, F_{t}\right)$, которые гладко меняются вместе с $t$. Напомним, что горазонтальные лифты hor ${ }^{\Gamma_{t}}$ и hor $^{\Gamma_{0}}$ являются пуассоновыми векторными полями 
для $\Lambda$ и, следовательно, hor ${ }^{\Gamma_{t}}-$ hor $^{\Gamma_{0}}$ является вертикальным пуассоновым векторным полем. Из предложения 2.6 следует, что существует зависящая от $t$ 1-форма $Q_{t} \in \Omega^{1}(B) \otimes \mathfrak{F}_{B}$ такая, что

$$
\operatorname{hor}^{\Gamma_{t}}(u)=\operatorname{hor}^{\Gamma_{0}}(u)+\Lambda^{\natural} d Q_{t}(u) .
$$

Для любого $t \in[0,1]$ имеем разложение

$$
T_{N} E=\mathbf{H}_{t} \oplus \mathbf{V},
$$

где $\mathbf{H}_{t}$ - горизонтальное подрасслоение, соответствующее связности $\Gamma_{t}$.

Пусть $\partial^{\Gamma_{t}}$ и $\partial^{\Gamma_{0}}$ - ковариантные внешние производные, соответствующие связностям $\Gamma_{t}$ и $\Gamma_{0}$ соответственно. В силу (3.3) имеем

$$
\partial^{\Gamma_{t}} \Theta=\partial^{\Gamma_{0}} \Theta+\left\{Q_{t} \wedge \Theta\right\}_{\Lambda}
$$

для $\Theta \in \Omega^{k}(B) \otimes \mathfrak{F}_{B}$.

Нас интересует следующее уравнение:

$$
L_{Z_{t}} \Pi_{t}+\frac{\partial \Pi_{t}}{\partial t}=0
$$

для зависящего от времени векторного поля $Z_{t}$ на $N$, обращающегося в нуль на $B$. В соответствии с (3.4) имеем разложение

$$
Z_{t}=X_{t}+Y_{t}
$$

где $X_{t}=X_{t}^{i}(\xi, x) \operatorname{hor}_{i}^{\Gamma_{t}}$ - это $\Gamma_{t}$-горизонтальное векторное поле и $Y_{t}=Y_{t}^{i}(\xi, x)\left(\partial / \partial x^{\sigma}\right)$ - вертикальное векторное поле.

ПрЕДЛОЖЕНИЕ 3.1. Зависящее от времени векторное поле $Z_{t}$ является решением уравнения (3.6) тогда и только тогда, когда

$$
\begin{gathered}
Y_{t}=\Lambda^{\natural} d h_{t}, \\
\partial^{\Gamma_{t}}\left(\boldsymbol{i}_{X t} F_{t}\right)+\left(\partial^{\Gamma_{t}}\right)^{2} h_{t}+\frac{\partial F_{t}}{\partial t}=0, \\
\boldsymbol{i}_{X_{t}} F_{t}=\frac{\partial Q_{t}}{\partial t}-\partial^{\Gamma_{t}} h_{t}+\beta_{t}
\end{gathered}
$$

для некоторых $h_{t} \in \mathfrak{F}_{B} u \beta_{t} \in \Omega^{1}(B) \otimes \mathfrak{C a s i m}_{B}(\Lambda)$.

Это утверждение доказывается непосредственным вычислением [9] с использованием предложения 3.1 и стандартных свойств скобки Схоутена и ковариантной внешней производной [14], [15].

В силу (3.3) для любого $t$ сужение $\partial^{\Gamma_{t}}$ на $\Omega^{k}(B) \otimes \mathfrak{C a s i m}_{B}(\Lambda)$ совпадает с кограничным оператором $\partial_{0}$. Рассмотрим $\partial_{0}$-коцикл Казимира $C_{t} \in \Omega^{2}(B) \otimes \mathfrak{C a s i m}_{B}(\Lambda)$, соответствующий паре $\left(\Pi_{t}, \Pi_{0}\right)$,

$$
C_{t}=F^{t}-F^{0}+\left(\partial^{\Gamma_{0}} Q_{t}+\frac{1}{2}\left\{Q_{t} \wedge Q_{t}\right\}_{\Lambda}\right) .
$$

Заметим, что $Q_{t}$ однозначно определяется условием (3.3) с точностью до преобразования $Q_{t} \mapsto Q_{t}+\kappa_{t}$ при $\kappa_{t} \in \Omega^{1}(B) \otimes \mathfrak{C a s i m}_{B}(\Lambda)$. Тогда $C_{t} \mapsto C_{t}+\partial_{0} \kappa_{t}$ и, следовательно, класс $\partial_{0}$-когомологий $\left[C_{t}\right]$ не зависит от выбора $Q_{t}$. 
СлеДСтвиЕ 3.2. Если уравнение (3.6) допускает решение $Z_{t}$, то

$$
\left[C_{t}\right]=0 \quad \forall t
$$

ДоКАЗАтЕЛЬСтво. Предположим, что условия (3.8)-(3.10) выполнены. Применяя оператор $\partial^{\Gamma_{t}}$ к обеим частям равенства (3.10), получаем

$$
\partial^{\Gamma_{t}}\left(\boldsymbol{i}_{X_{t}} F_{t}\right)+\left(\partial^{\Gamma_{t}}\right)^{2} h_{t}=\partial^{\Gamma_{t}}\left(\frac{\partial Q_{t}}{\partial t}\right)+\partial^{\Gamma_{t}} \beta_{t} .
$$

Подстановка этого тождества в (3.9) дает

$$
\partial^{\Gamma_{t}}\left(\frac{\partial Q_{t}}{\partial t}\right)+\partial^{\Gamma_{t}} \beta_{t}+\frac{\partial F_{t}}{\partial t}=0
$$

Из (3.5) следует, что

$$
\partial^{\Gamma_{t}} \beta_{t}=\partial^{\Gamma_{0}} \beta_{t}=\partial_{0} \beta_{t}, \quad \partial^{\Gamma_{t}}\left(\frac{\partial Q_{t}}{\partial t}\right)=\frac{\partial}{\partial t}\left[\partial^{\Gamma^{0}} Q_{t}+\frac{1}{2}\left\{Q_{t} \wedge Q_{t}\right\}_{\Lambda}\right] .
$$

Применяя эти формулы, выводим из (3.13), что

$$
\frac{\partial F_{t}}{\partial t}=-\frac{\partial}{\partial t}\left[\partial^{\Gamma_{0}} Q_{t}+\frac{1}{2}\left\{Q_{t} \wedge Q_{t}\right\}_{\Lambda}\right]-\partial^{\Gamma_{0}} \beta_{t}
$$

и, следовательно,

$$
C_{t}=-\partial_{0}\left(\int_{0}^{t} \beta_{\tau} d \tau\right)
$$

Это доказывает (3.12).

СлЕДСтвиЕ 3.3. Предположим, что (3.12) выполняется. Тогда уравнение (3.6) допускает решение $Z_{t}$.

ДокАЗАтЕЛЬСтво. Если (3.12) выполняется, то можно выбрать $Q_{t}$ в (3.3) так, чтобы $C_{t}=0$. Отсюда следует, что

$$
\frac{\partial F_{t}}{\partial t}=-\left[\partial^{\Gamma_{0}}\left(\frac{\partial Q_{t}}{\partial t}\right)+\left\{Q_{t} \wedge \frac{\partial Q_{t}}{\partial t}\right\}_{\Lambda}\right]=-\partial^{\Gamma_{t}}\left(\frac{\partial Q_{t}}{\partial t}\right)
$$

Тогда условие (3.9) принимает вид

$$
\partial^{\Gamma_{t}}\left(\boldsymbol{i}_{X t} F_{t}\right)+\left(\partial^{\Gamma_{t}}\right)^{2} h_{t}-\partial^{\Gamma_{t}}\left(\frac{\partial Q_{t}}{\partial t}\right)=0 .
$$

В силу невырожденности $F_{t}$ существует единственное $\Gamma_{t}$-горизонтальное векторное поле $X_{t}$ такое, что

$$
\boldsymbol{i}_{X t} F_{t}=\frac{\partial Q_{t}}{\partial t}
$$

Полагая $h_{t}=0, \beta_{t}=0$ и $Z_{t}=X_{t}$, мы видим, что уравнения (3.8)-(3.10) удовлетворяются. 
ДоКАЗАТЕЛЬСТВо тЕОРЕМЫ 2.7. Заметим, что для любых двух тензоров спаривания $\Pi^{\prime}$ и $\Pi^{\prime \prime}$ в $\mathfrak{C o u p}_{B}(E, \Lambda)$ можно определить относительный коцикл Казимира $C\left(\Pi^{\prime}, \Pi^{\prime \prime}\right)$ с помощью (2.35). При этом имеем следующие свойства:

$$
\begin{gathered}
{\left[C\left(\Pi^{\prime}, \Pi^{\prime \prime}\right)\right]=-\left[C\left(\Pi^{\prime \prime}, \Pi^{\prime}\right)\right],} \\
{\left[C\left(\Pi^{\prime}, \Pi^{\prime \prime}\right)\right]+\left[C\left(\Pi^{\prime \prime}, \Pi^{\prime \prime \prime}\right)\right]+\left[C\left(\Pi^{\prime \prime \prime}, \Pi^{\prime}\right)\right]=0 .}
\end{gathered}
$$

Зафиксируем экспоненциальное отображение $\boldsymbol{f}_{0}$ и диффеоморфизм $g$ в $(2.32)$ и рассмотрим пуассонов тензор спаривания $\widetilde{\Pi}=\left(\boldsymbol{f}_{0} \circ g\right)^{*} \Psi$, соответствующий данным $(\Lambda, \widetilde{\Gamma}, \widetilde{F})$. Предположим, что задан диффеоморфизм $\varphi \in \mathfrak{G}_{B}(E)$ такой, что $\left(\varphi^{*} \widetilde{\Pi}\right)_{V}=\Lambda$. Заметим, что из последнего условия не следует, что $\varphi$ сохраняет слои и является автоморфизмом для $\Lambda$. Таким образом, $\varphi^{*} \widetilde{\Pi} \in \mathfrak{C o u p}_{B}(E, \Lambda)$, и мы должны показать, что

$$
\left[C\left(\widetilde{\Pi}, \Pi^{(1)}\right)\right]=\left[C\left(\varphi^{*} \widetilde{\Pi}, \Pi^{(1)}\right)\right] .
$$

Для каждого $t \in[0,1]$ рассмотрим послойно сохраняющее отображение

$$
m_{t}: E \rightarrow E, \quad m_{t}(\xi, x)=(\xi, t x) .
$$

Можно предположить, что $\varphi$ корректно определен в окрестности $N$ листа $B$ в $E$ так, что $N \cap E_{\xi}$ является звездной областью при любом $\xi \in B$. Рассмотрим семейство диффеоморфизмов $\Phi_{t}=m_{t}^{-1} \circ \varphi \circ m_{t} \in \mathfrak{G}_{B}(E)$. Имеем $\Phi_{0}=\mathrm{id}, \Phi_{1}=\varphi$. Далее, $\left.\Phi_{t}\right|_{B}=\mathrm{id}_{B}$ и, следовательно, можно выбрать окрестность $N$ листа $B$ в $E$ так, чтобы $\Phi_{t}$ было корректно определено на $N$ при всех $t \in[0,1]$. Следовательно, $\Phi^{t}$ является фазовым потоком зависящего от времени векторного поля

$$
Z_{t}=\left(\frac{d \Phi_{t}}{d t}\right) \circ \Phi_{t}^{-1}
$$

обращающегося в нуль на $B$. Рассмотрим гладкую кривую

$$
t \mapsto \Pi_{t}=\left(\Phi^{t}\right)^{*} \widetilde{\Pi} \in \mathfrak{C o u p}_{B}(E, \Lambda),
$$

соединяющую $\widetilde{\Pi}$ и $\varphi^{*} \widetilde{\Pi}$. Ясно, что $Z_{t}$ в (3.17) является решением (3.6). Тогда в силу следствия 3.2 условие (3.12) выполняется. В частности, $\left[C_{1}\right]=\left[C\left(\varphi^{*} \widetilde{\Pi}, \widetilde{\Pi}\right)\right]=0$. Наконец, применяя свойство (3.15), мы получаем (3.16).

ДоКАЗАТЕЛЬСтво теОРемЫ 2.8. Достаточность. Предположим, что (2.36) выполнено. Тогда можно выбрать $Q \in \Omega^{1}(B) \otimes \mathfrak{F}_{B}$ в $(2.34)$ так, чтобы получить $C=0$. Нужно построить диффеоморфизм $\varphi$, удовлетворяющий условию $\varphi^{*} \widetilde{\Pi}=\Pi^{(1)}$. Для любого $t \in[0,1]$ определим связность Эресмана $\Gamma_{t}=\Gamma-t \Lambda^{\sharp} d Q$ и 2-форму

$$
F_{t}=F^{(1)}-\left(t \partial^{\Gamma^{(1)}} Q+\frac{t^{2}}{2}\{Q \wedge Q\}_{\Lambda}\right) .
$$

Стягивая при необходимости окрестность $N$ листа $B$, мы можем считать, что для любого $t$ форма $F_{t}$ является горизонтально невырожденной (условие (2.7)). Можно показать, что данные $\left(\Lambda, \Gamma^{t}, F^{t}\right)$ удовлетворяют соотношениям $(2.16)-(2.19)$ и, следовательно, порождают зависящий от времени пуассонов тензор спаривания $\Pi_{t}$, который определяет гладкий путь в $\mathfrak{C o u p}_{B}(E, \Lambda)$, соединяющий П(1) и $\widetilde{\Pi}$. Из (3.18) 
следует, что $C_{t}=0$. Применяя следствие 3.3 , заключаем, что существует зависящее от времени векторное поле $X_{t}=X_{t}^{i}(\xi, x)$ hor $_{i}^{\Gamma_{t}}$, удовлетворяющее (3.6). В нашем случае $X_{t}$ однозначно определяется уравнением $i_{X t} F^{t}=Q$. Так как $X_{t}$ обращается в нуль вдоль $B$, можно снова стянуть $N$ так, чтобы поток $\Phi^{t}$ поля $X_{t}$ был корректно определен на $N$ для всех $t \in[0,1]$. Таким образом, $\left(\Phi^{t}\right)^{*} \Pi_{t}=\Pi_{0}$ и можно положить $\varphi=\Phi^{1}$.

Необходимость следует из теоремы 2.7.

Доказательство теоремы 2.10 проводится аналогично.

Автор благодарен М. В. Карасеву за полезные обсуждения при подготовке этой статьи.

\section{СПИСОК ЦИТИРОВАННОЙ ЛИТЕРАТУРЫ}

[1] В. И. Арнольд, Математические методы классической механики, Наука, М., 1974.

[2] A. Weinstein, "The local structure of Poisson manifolds", J. Differential Geom., 18:3 (1983), 523-557.

[3] J. Conn, "Normal forms for smooth Poisson structures", Ann. of Math. (2), 121:3 (1985), $565-593$.

[4] J.-P. Dufour, "Linéarisation de certaines structures de Poisson", J. Differential Geom., 32:2 (1990), 415-428.

[5] N. T. Zung, arXiv: math.SG/0207263, 2002.

[6] R. L. Fernandes, P. Monnier, "Linearization of Poisson brackets", Lett. Math. Phys., 69 (2004), 89-114.

[7] Yu. Vorobjev, "Coupling tensors and Poisson geometry near a single symplectic leaf", Lie Algebroids and related topics in differential geometry, Banach Center Publ., 54, Warszawa, 2001, 249-274.

[8] Ю. М. Воробьев, "О линеаризованных пуассоновых структурах", Матем. заметки, 70:4 (2001), 486-493.

[9] Yu. Vorobjev, arXiv: math.SG/0503628v1, 2005.

[10] I. Vaisman, "Coupling Poisson and Jacobi structures on foliated manifolds", J. Geom. Method. Mod. Phys., 1:5 (2004), 607-637.

[11] B. L. Davis, A. Wade, "Nonlinearizability of certain Poisson structures near a symplectic leaf", E-print math.SG/0406611, 2005.

[12] O. Brahic, arXiv: math.SG/0403136, 2004.

[13] M. Crainic, R. L. Fernandes, arXiv: math.DG/0503145v1, 2005.

[14] М. В. Карасев, В.П. Маслов, Нелинейные скобки Пуассона. Геометрия и квантование, Наука, М., 1991.

[15] I. Vaisman, Lectures on the Geometry of Poisson Manifolds, Progress in Math., 118, Birkhäuser, Boston, 1994. 